\title{
A computer vision approach for weeds identification through Support Vector Machines
}

\author{
Alberto Tellaeche $^{\mathrm{a}, *}$, Gonzalo Pajares ${ }^{\mathrm{a}, 1}$, Xavier P. Burgos-Artizzu ${ }^{\mathrm{b}}$, Angela Ribeiro ${ }^{\mathrm{b}}$ \\ a Dpto. Ingeniería del Software e Inteligencia Artificial, Facultad Informática, Universidad Complutense, Madrid 28040, Spain \\ b Instituto de Automática Industrial, CSIC, Arganda del Rey, Madrid, Spain
}

\begin{abstract}
A B S T R A C T
This paper outlines an automatic computer vision system for the identification of avena sterilis which is a special weed seed growing in cereal crops. The final goal is to reduce the quantity of herbicide to be sprayed as an important and necessary step for precision agriculture. So, only areas where the presence of weeds is important should be sprayed. The main problems for the identification of this kind of weed are its similar spectral signature with respect the crops and also its irregular distribution in the field. It has been designed a new strategy involving two processes: image segmentation and decision making. The image segmentation combines basic suitable image processing techniques in order to extract cells from the image as the low level units. Each cell is described by two area-based attributes measuring the relations among the crops and weeds. The decision making is based on the Support Vector Machines and determines if a cell must be sprayed. The main findings of this paper are reflected in the combination of the segmentation and the Support Vector Machines decision processes. Another important contribution of this approach is the minimum requirements of the system in terms of memory and computation power if compared with other previous works. The performance of the method is illustrated by comparative analysis against some existing strategies.
\end{abstract}

\section{Introduction}

In these days, there is a clear tendency of reducing the use of chemical agents in agricultural cultivations. The main goals of all the techniques developed towards this objective try to obtain products of a better quality and the saving of costs related to the crop field treatments. This tendency has been established over the recent years among various countries, creating a growing interest [1].

The development of computer vision capabilities allows a reliable and fast identification and classification of seeds. Automatic systems nowadays provide techniques to easily process the crop fields to obtain the necessary data to classify and to distinguish the crop from the weeds. The developments of these systems are mainly based on the computation of geometrical characteristics of the weeds because they have forms (shape factor, aspect ratio, length/ratio, etc.) which can be identified [2-5]. Colour images have been also successfully used to establish seed quality and characterize damages and diseases [6]. Yang et al. [7] estimated weed coverage and weed patchiness based on digital images, using a fuzzy algorithm for planning site-specific herbicide applications. Recently, Gerhards and Oebel [8] have used real-time differential images (NIR-VIS) obtained with a set of 3 digital bispectral cameras. Other approaches have used colour indices to distinguish vegetation material from background [9].

Unlike the above approach our method tries to identify and classify a special weed seed, the avena sterilis ssp. Ludoviciana, which is one of the most widely distributed and abundant weed of cereals in Spain and other regions with Mediterranean climate and causes substantial losses in these crops $[10,11]$. The main problem concerning its classification is that during the herbicide treatment its colour and texture are undistinguishable with respect to the seed cereal (barley). In Ref. [12] an image segmentation process is carried out by taking into account the row-spacing in the crops, which allows driving the process. The avena sterilis grows without control along the field, it can be found mixed with the cereal in the crop lines and also out of the crop lines in the soil where the cereal does not grow.

Hence, the above techniques cannot be applied because of the similar spectral aspect and texture and also its irregular shapes and distributions. Based on this behaviour and taking into account the expertise of technical people and farmers, it has been formulated the following hypothesis: "a high density of green colour in soil areas where the cereal is assumed to be missing indicates that this region, including the crop lines, is contaminated with avena 
sterilis". Indeed, if the soil has seeds, they probably are avena and the surrounding crop cereal areas will also contain this weed.

The design of a system to perform a selective spraying of the crops can help to minimize the wastage of products required for the effective control of weeds, diseases and pests and to ensure that crops receive adequate nutrients [13]. In this context, the benefit of site-specific management includes a cost reduction to produce the crop and also an important reduction in environmental pollution [14].

From the above, several systems have been designed to solve this problem using automatic vision strategies and various machine learning techniques to identify areas with avena sterilis which must be selectively sprayed [15-21]. Despite the good performance of these systems, all of them share a common inconvenient, the techniques used to take a decision are very demanding from a computational point of view. Also, all these methods require a considerable amount of memory to deal with all the information necessary for the decision making process. Because of these constraints the real implementation of the systems previously developed can require an expensive hardware.

As a further approach, this new research outlines a new decision making method, the Support Vector Machines (SVMs) whose two main advantages are a fast decision computation once trained and an optimized storage of knowledge database, as explained later in Section 2.2.3. Additional advantages of SVMs are also given in Section 2.2.4.

As in previous attempts, two main processes are involved in our approach: image segmentation and decision making. The image segmentation process divides the crop field in areas to be analysed and extracts features and attributes of the selected areas. The decision making process determines which areas are to be selectively sprayed.

The features are extracted by using image regions and line segmentation techniques, which provide a set of patches. According to the distribution of these patches they can be identified as representing weeds or crops. The attributes are computed taking into account area-based relations between the crops, the weeds and the soil. Relative measurements (relations) have been used because of the irregular forms of weeds and crops, and also due to the different size of the cells resulting from the segmentation process as it can be seen later. Once the attributes are quantified, the SVM approach is used for making the decision about the spraying of a cell. The design of a new automatic image vision approach that integrates the segmentation and the SVM decision making approach as an optimized method by means of computation and knowledge storage makes the main finding of the proposed approach.

The paper is organized as follows: Section 2 describes the weed detection process including the image segmentation and the SVM decision making one. The performance of this approach is described in Section 3. Finally in Section 4 the conclusions are presented.

\section{The weed detection process}

The full weed detection process consists of two sub-processes: image segmentation and decision making. The image segmentation process divides the incoming image in cells and extracts features and attributes, which are used during the decision making process. The decision process determines if a cell is to be or not sprayed. Previous to the decision making process, a database containing a set of samples classified as items to be or not sprayed is built. Hence, two separated processes can be distinguished: off-line and on-line. During the off-line a knowledge base $(K B)$ is built so that a decision can be made during the on-line process by recovering the information from $K B$. The image segmentation process is identical for both off-line and on-line processes. Fig. 1 displays the full sequence.

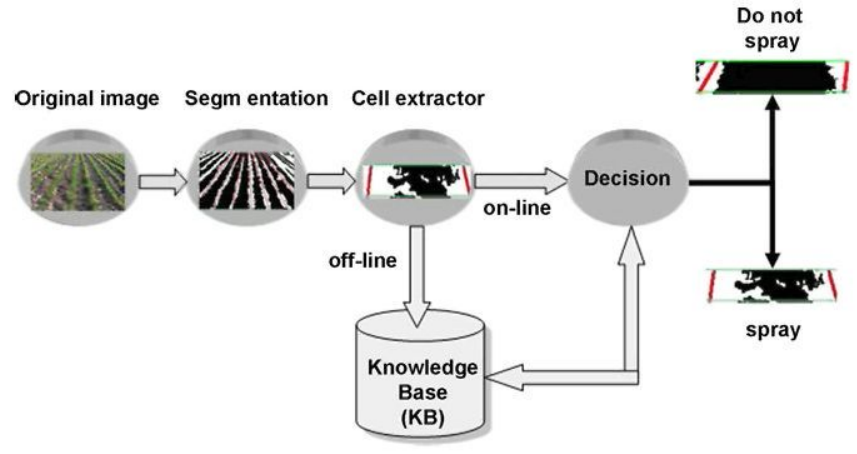

Fig. 1. Vision-based decision process.

\subsection{Image segmentation}

The steps involved in the image segmentation process are: image acquisition and binarization, crop lines detection, grid cell partition, attribute extraction. In Ref. [12] a segmentation process is proposed under the knowledge of row distances. The RGB original image is binarized by applying a threshold to the grey image obtained from the RGB. The frequency of the plant pixels is plotted in the crop row direction; a maximum value indicates a row. After morphology dilation the weeds appear isolated and can be identified. In our approach the avena sterilis do not present this behaviour. This justifies the definition of a new approach for detecting this special kind of weeds in cereal crops.

\subsubsection{Image acquisition and binarization}

The images are acquired under perspective transformation, Fig. 2. This implies that the crop lines tend to converge in the

(a)

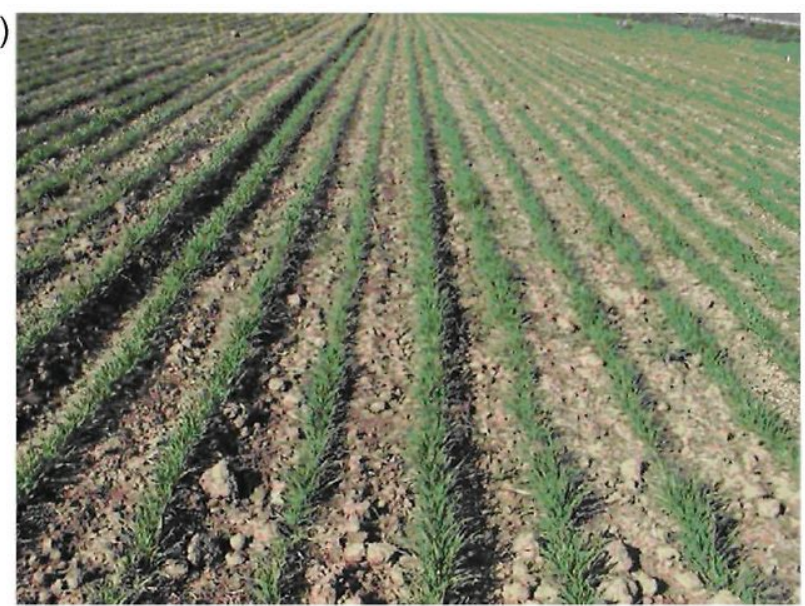

(b)

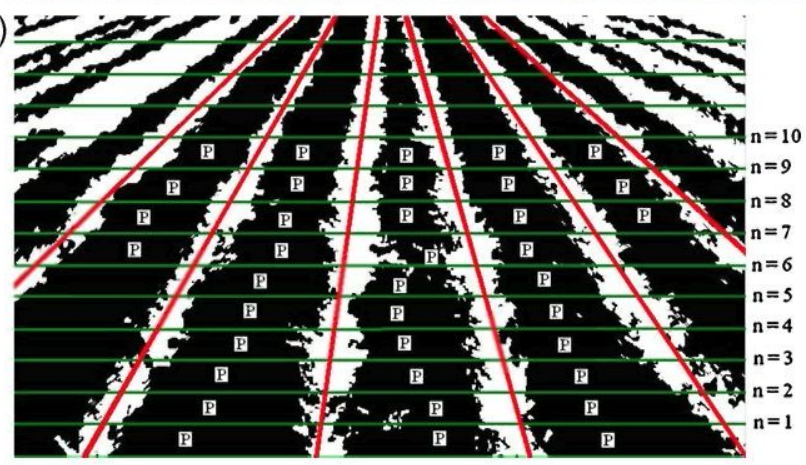

Fig. 2. (a) Original image and (b) segmented image displaying the line crops and cells. 
vanishing point out of the field of view. The goal of this first step is to convert the input red-green-blue image into a black and white image, where the vegetation parts (weed and crops) of the input image are to be represented as white and the rest as black in a binary image.

Some approaches have been proposed to do that (Ribeiro, 2005 [29]; Granitto, 2005 [4]; Onyango, 2003 [5]; Tian, 1998 [22]). Based on the discussion carried out in Ribeiro (2005) this method has been used. The segmentation is based on the three components (RGB) that describe each image point. The first stage of the segmentation transforms the original RGB image into a one-dimensional grey level (monochrome) image, obtained by applying the following expression:

$T(i, j)=r R(i, j)+g G(i, j)+b B(i, j)$

where $r, g$ and $b$ are the set of real coefficients to be selected, and whose possible values are discussed in Ribeiro (2005), where the best performance is achieved with the following values: $r=-1, g=2$ and $b=-1$; if $T(i, j) \leq 0$ then $T(i, j)=0$; if $T(i, j) \geq 255$ then $T(i, j)=255$, i.e. the grey level output values range in $[0,255]$.

The next step is to determine the grey level threshold that sets the contrast breakpoint between pixels containing vegetation and pixels containing non-vegetation, including shadows, stones, straw, and other debris, and then to transform the grey level image into a black/white image to obtain a binary image. In Rosin and Ioannidis [23] are evaluated several classical global image thresholding approaches. Based on this study, the best performance is achieved with the method described in Kapur et al. [24], which uses the entropy of the histogram. This approach is used.

Once the image is binarized, in order to remove spurious white pixels and to smooth white contours it is applied a morphological opening (erosion followed by dilation) operation. This kind of operation is also used in Onyango and Marchant [5]. Nevertheless, making use of the knowledge that the image is acquired under perspective transformation, there are applied three different structuring elements for performing the morphological opening operation. Indeed the central line crops are near vertical, but in the pieces of images to the left and right of the central image, the line crops have different slopes. The image is divided in three parts with the same width: left $(L)$, central $(C)$ and $(R) . S_{L}, S_{C}$ and $S_{R}$ are used as structuring elements in (2) to be applied in the $L, C$ and $R$ parts, respectively.

$S_{L}=\left[\begin{array}{lllll}0 & 0 & 0 & 1 & 1 \\ 0 & 0 & 1 & 1 & 1 \\ 0 & 1 & 1 & 1 & 0 \\ 1 & 1 & 1 & 0 & 0 \\ 1 & 1 & 0 & 0 & 0\end{array}\right], \quad S_{L}=\left[\begin{array}{lllll}0 & 1 & 1 & 1 & 0 \\ 0 & 1 & 1 & 1 & 0 \\ 0 & 1 & 1 & 1 & 0 \\ 0 & 1 & 1 & 1 & 0 \\ 0 & 1 & 1 & 1 & 0\end{array}\right]$,

$S_{R}=\left[\begin{array}{lllll}1 & 1 & 0 & 0 & 0 \\ 1 & 1 & 1 & 0 & 0 \\ 0 & 1 & 1 & 1 & 0 \\ 0 & 0 & 1 & 1 & 1 \\ 0 & 0 & 0 & 1 & 1\end{array}\right]$

\subsubsection{Crop lines detection and grid cell partition}

In the resulting binary image, after the opening operation, plant material from both weeds and crops is white and the rest, coming from soil, stones and residual is black. On the basis of the binary image the next step is to detect the furrows of the crops in the image. The Hough transform is a well-known and robust method, especially if the lines cover the whole image as reported in Astrand and Baerveldt [14] and Billingsley and Schoenfisch [25].

The Hough transform is a common operation to obtain line equations that define a group of points in the $x y$ space of the image $[26,27]$. Instead of using the parameter space $x y$, the normal representation of a line: $x \cos \theta+y \sin \theta=\rho$ is used. This allows considering the full range of values for $\theta=-90^{\circ}$ to $\theta=90^{\circ}$ avoiding the problem of selecting the range of the parameters in the parameter space.

The Hough transform creates an accumulator of cells $A(\theta, \rho)$ indexed by $\theta$ and $\rho$. At the end of the Hough transform application, a value of $Q$ in a given cell $A(\theta, \rho)$ means that $Q$ points in the original image lie in the line $x \cos \theta+y \sin \theta=\rho$. The full image is used to extract high $Q$ values. This allows us to remove cells with small $Q$ values that do not represent crop lines. The cells with values less than the threshold $T_{h}$ are removed. $T_{h}$ is set to 100 in this paper. Moreover, as it is known from the perspective transformation, the orientation of the crop lines is limited, the lines searched must be coherent with the knowledge of $\theta$ and $\rho$. Finally, because the crops have a wide dimension, it is useful that several accumulator cells with similar indices $(\theta$ and $\rho$ ) have high $Q$ values. This means that several lines are associated to the same crop. All similar ones are fused in a unique one. So given two cells $A\left(\theta_{i}, \rho_{i}\right)$ and $A\left(\theta_{j}, \rho_{j}\right)$, it is assumed that they represent the same line crop if $\left|\theta_{i}-\theta_{j}\right| \leq \varepsilon_{1}$ and $\left|\rho_{i}-\rho_{j}\right| \leq \varepsilon_{2}$ where $\varepsilon_{1}$ and $\varepsilon_{2}$ are set to 5 and 10 , respectively.

The next step consists in the partition of the image in cells. This is carried out by tracing horizontal lines, those lines are described through equations with the form: $y=k c$, where the joint product $k c$ is a constant value; $k$ represents an index varying from 1 to $n$ and $c$ determines the spacing between lines. We have fixed $c=50$ and $n$ is limited by the height of the image. Nevertheless, because we are only interested on the set of cells on the lower part of the image $n$ is fixed to $\mathbf{1 0}$ in our experiments. This interest comes as a result of the perspective projection, which causes that the size of the cells decreases towards the upper part of the image and an important amount of details coming from weeds are lost when more cells are used. It has been considered acceptable a number between 35 and 37 cells, depending on the image system position at the instant of the capture. They are the cells where the weeds are distinguishable because of their proximity to the vision system. Fig. 2(a) shows an original image captured in a crop field; in (b) is displayed the resulting image segmentation process, including the crop's lines and cells. The 37 cells to be processed have been labelled in this image with the symbol " $P$ ". To avoid problems during the decision process, these are the cells which appear full in the image, i.e. perfectly delimited by the horizontal lines and the lines describing the furrows. We have not considered incomplete cells to avoid problems during the decision process.

\subsubsection{Attribute extraction}

The basic unit to be analysed is the cell. Due to the perspective transformation the shape and size of the cells differ among them. Some existing strategies have been used for identifying the weeds in crop fields. Granitto et al. [4] and Astrand and Baerveldt [14] use topological properties (area, invariant moments, etc.); colour (variance, skewness of the intensity histogram, etc.) or texture. Unfortunately, the weed seeds which are to be identified in our approach (avena sterilis) appear in patches under irregular sizes and shapes. The colour and texture for this weed are similar to that of the cereal in the field. Hence, the above attributes are not applicable in our approach.

Moreover, this kind of weed grows uncontrolled in the field. This means that white patches in soil areas between crops should be avena sterilis and the surrounding crop areas are probably affected by weed seeds. This represents a serious handicap when a decision must be made about if the cell must be sprayed. This irregular cereal distribution is affected by the large drought periods in the Spanish central area where this experience has been carried out. Indeed, some areas in the furrows display important gaps against other that invade the soil between furrows. Additionally, given the image 
perspective projection the cells are different in size and shape. To solve these problems, attributes extracted must be independent of the distribution of weeds and crops and also from the size and shape of the cells. This requires the computation of relative measurements instead of absolute ones.

With such purpose a set of 20 images have been randomly selected to be segmented from a set of 86 images available, see Section 3. From each segmented image, as mentioned before, between 35 and 37 cells are selected, on average we have obtained 36 cells per image; i.e. the amount of cells is 720 . The number of cells classified as candidate to be sprayed is $F Y_{0}=156$, i.e. this represents the $21.7 \%$ of 720 . This relative small percentage reinforces the interest for selective spraying. From the remainder set of cells (i.e. $F N_{0}=564$ ), the ratio of the white area in the cell is computed,

$r=\frac{1}{F N_{0}} \sum_{c=1}^{F N_{0}} \frac{W_{c}}{A_{C}}$

$A_{c}$ is the full area of a cell $c$ and $W_{c}$ is the white area in the cell. In this kind of cells, free of weeds, the white area represents only crops. Each cell contains left $(L)$ and right $(R)$ patches representing the crop areas. It can be found that $r \approx 2 / 5$ and $r=r_{l}+r_{r}$ where $r_{1}$ and $r_{r}$ are the corresponding ratios for the $L$ and $R$ crop areas, respectively. This means that $r_{t}=r_{\mathrm{r}} \approx 1 / 5$, i.e. each crop area is covering $1 / 5$ of the full cell's area.

Two area-based attributes are computed and embedded as the components of an area-vector $\mathbf{x}_{i}$, as before given the cell $i$, this vector is $x_{i}=\left\{x_{i 1}, x_{i 2}\right\}$. Let $m$ the total number of connected regions in a cell $i$ (i.e. the number of labels in the cell) and $A_{i j}$ the area of the $j$ th region. $A_{i c}$ is the total area of the cell. $A_{i L}$ and $A_{i R}$ the areas for the $L$ and $R$ crop regions, respectively. $A_{i L}$ and $A_{i R}$ are computed taking into account the amount of pixels inside of the regions bounded by the left and right crop lines respectively and the corresponding limits defined by $r_{l}$ and $r_{r}$ (i.e. $1 / 5$ of $A_{i c}$ ).

Based on the area measurements the following coverage values are computed, crop coverage:

- crop coverage:

$$
C_{i c}=A_{i L}+A_{i R}
$$

- weed coverage:

$$
C_{i w}=\sum_{j=1}^{m} A_{i j}-C_{i c}
$$

- soil coverage:

$$
C_{i S}=A_{i C}-\left(C_{i c}+C_{i w}\right)
$$

From Eqs. (4)-(6) the components for the area-vector $x_{i}$ are

$x_{i 1}=\frac{C_{i w}}{A_{i c}}$ and $\quad x_{i 2}=\frac{C_{i w}}{C_{i c}}\left(1-\frac{C_{i s}}{A_{i c}}\right)$

The component $x_{i 1}$ is defined as the weed coverage rate in Tian et al. [28] and $x_{i 2}$ can be associated with the weed pressure defined also in Ribeiro et al. [29].

The following analysis allows us to determine the range of variability for these two values. Indeed, if the weed coverage is null, there is not weeds in the cell, i.e. $x_{i 1}$ and $x_{i 2}$ are both null; but if the weeds cover the full intermediate region (i.e. $C_{i w}=(3 / 5) A_{i c}$ ) then $a_{i 1}=3 / 5$. Hence, $x_{i 1}$ ranges in $[0,3 / 5]$. The upper limit of $x_{i 2}$ is achieved when $C_{i w}$ is maximum (i.e. $C_{i w}=(3 / 5) A_{i c}$ ) and $C_{i c}$ minimum (i.e. $C_{i c}=0$ ); but if $C_{i c}$ is null this means that the cell has not crops. This special case has not been found in the experiments. The minimum value obtained for $C_{i c}$ was $(1 / 10) A_{i c}$. Now, assuming that $C_{i w}=(3 / 5) A_{i c}$, then $C_{i s}=0.3 A_{i c}$. Finally, the upper limit for $x_{i 2}$ can be fixed from Eq. (6) as 4.2. Based on these limits, the component values of the area-vector are mapped linearly to range both in $[0,1]$. This is intended so that both components contribute equitably in the computation of the decision function.

\subsection{Support Vector Machines as a decision making process}

Our system works in two stages: (1) performing a training process, called off-line, with a set of cells requiring to be sprayed and not to be sprayed, and computing a decision function; (2) performing a decision making process, called on-line, where a decision is made about each new incoming cell based on the above function.

The segmentation process is common for both off-line and on-line processes. This means that the cells and attributes are computed in identical fashion.

\subsubsection{The off-line process: training}

The SVM framework is based on the observation of a set $X$ of $n$ patterns samples (cells in our approach) to be classified as cells to be sprayed $(F Y)$ and not sprayed $(F N)$, i.e. our problem is the well-known binary classification problem. Theoretically, the outputs of the system are two symbolic values $y \in\{+1,-1\}$ associated to $F Y$ and $F N$, respectively. The finite training set is denoted by $\left(\mathbf{x}_{i}\right.$, $\left.y_{i}\right), i=1, \ldots, n$. where each $x_{i}$ vector denotes a training element and $y_{i} \in\{+1,-1\}$ the class it belongs to. The goal of this training process, based on the information stored in the training sample set, is to find a decision function capable of separating the data into two groups. It has been verified that the $x_{i}$ input vectors belong to two overlapped classes. The technique is based on the idea of mapping the input vectors into a high dimensional feature space using non-linear transformation functions. In the feature space a separating hyperplane (a linear function of the attribute variables) is constructed [30,31]. By using different mapping functions, different types of SVM are implemented. The SVM decision function has the general form,

$f(\mathbf{x})=\sum_{i=1}^{n} \alpha_{i} y_{i} H\left(x_{i}, \mathbf{x}\right)-b$

where $b$ is a constant; $\mathbf{x}$ is a generic two-dimensional area-vector representing the attributes of a generic cell, where its components are obtained through Eq. (7).

Eq. (8) establishes a representation of the decision function $f(\mathbf{x})$ as a linear combination of kernels centred in each data point. A discussion about the choice of the kernel used in this approach is given in Section 3.2.

The parameters $\alpha_{i}, i=1, \ldots, n$, in Eq. (8) are the solution for the following quadratic optimization problem:

Maximize the functional

$$
\begin{aligned}
& Q(\alpha)=\sum_{i=1}^{n} \alpha_{i}-\frac{1}{2} \sum_{i, j=1}^{n} \alpha_{i} \alpha_{j} y_{i} y_{j} H\left(x_{i}, x_{j}\right) \sum_{i=1}^{n} y_{i} \alpha_{i}=0 \\
& 0 \leq \alpha_{i} \leq \frac{c}{n}, i=1, \ldots, n
\end{aligned}
$$

and given the training data $\left(x_{i}, y_{i}\right), i=1, \ldots, n$, the inner product kernel $H$, and the regularization parameter $C$. As stated in Cherkassky and Mulier [30], at present, there is not a well-developed theory on how to select the best $C$, although in several applications it is set to

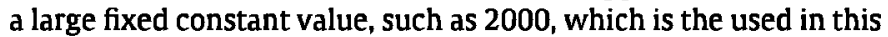
paper.

The data points $\mathbf{x}_{i}$ associated with the nonzero $\alpha_{i}$ are called support vectors. Once the support vectors have been determined, the 
SVM decision function has the form

$f(\mathbf{x})=\sum_{\text {support vectors }} \alpha_{i} y_{i} H\left(\mathbf{x}_{i}, \mathbf{x}\right)-b$

In the experiments, $b=0$; where $\mathbf{x}$ is defined in Eq. (8).

The SVM generates a scalar output $f(x)$ whose polarity, sign of $f(\mathbf{x})$, determines the class membership. The magnitude can usually be interpreted as a measure of belief or certainty in the decision made. If all data points which are not support vectors were to be discarded for the training set the same solution would be found, an interesting perspective on SVMs is to consider its information compression and storage properties. The support vector represent the most informative data points and compress the information contained in the training set, i.e. in classification approaches, only the support vectors need to be stored, while all other training examples can be discarded [32].

The minimal distance from the separating hyperplane to the closest data point is called margin $\tau$ [30]. A separating hyperplane is called optimal if the margin is maximum. The distance between the separating hyperplane and a given pattern $\mathbf{x}$ is $y_{k} f(\mathbf{x})|/||\mathbf{w}| \mid$ where $w$ is given by

$w=\sum_{i=1}^{n} \alpha_{i} y_{i} x_{i}$

Assuming that a margin $\tau$ exists, all training patterns obey the inequality,

$\frac{y_{k} f\left(\mathbf{x}_{k}\right)}{\|\boldsymbol{w}\|} \geq \tau, \quad k=1, \ldots, n$ where $y_{k}=\{+1,-1\}$

The problem of finding the optimal hyperplane is that of finding the $\mathbf{w}$ that maximizes the margin $\tau$. Note that there are an infinite number of solutions that differ only in scaling of $\mathbf{w}$. To limit solutions, fix the scale on the product of $\tau$ and norm of $\mathbf{w}$,

$\tau|| \boldsymbol{w}||=1$

Thus maximizing the margin $\tau$ is equivalent to minimizing the norm of $\mathbf{w}$.

\subsubsection{The on-line process: decision making}

Now given a new image it can be applied the segmentation process described in Section 2.1, i.e. for each Image 36 cells can be obtained. For each cell $k$ the attribute's vector $\mathbf{x}_{k}$ is computed as well as $f\left(x_{k}\right)$ through Eq. (10) by recovering the support vectors stored during the off-line process. According to the sign of $f\left(x_{k}\right)$ the cell which is being processed should be sprayed if $f\left(x_{k}\right)>0$ (it belongs to $F Y$ ) otherwise it should not be sprayed.

\subsubsection{Computation complexity and knowledge information} storage

As stated before in this article, in this case, the selection of the pattern recognition algorithm has been carried out taking into account the computational resources required by it for making a decision. This has been taken in mind with the aim of minimizing the price of the hardware needed and the price of the final decision making system.

From this point of view, the selection of the Support Vector Machines approach can be justified by two main reasons:

1. Although the learning process can be time consuming, once the support vectors are obtained, the decision is made by means of Eq. (10). This equation is composed only by elemental arithmetical operations (sum, subtraction and multiplications), thus, the decision can be computed in a very fast way with very simple processors.

2. The second advantage that this approach show is the minimum memory requirements to store the knowledge obtained from the training process. Because of the nature of the classifier, it is only necessary to store the support vectors obtained after the training process. The number of these vectors is really small when compared with the totality of cases processed, but they store all the valuable information. Any other vector of information stored apart from the support vectors is redundant.

\subsubsection{Advantages of SVMs for the bi-class spraying decision problem}

As it is well-known in the learning community, SVM is a technique well tested in binary classification problems, as the one proposed here. All, classification methods have advantages and disadvantages, which are more or less important according to the data which are being analysed. In our selective spraying problem SVMs are useful because of non-regularity in the data, i.e. with an unknown distribution. The following points include a discussion about the SVMs performance in our approach:

1. By introducing the kernel, SVMs are able to choose the threshold separating areas to be sprayed, which need not be linear.

2. Since the kernel implicitly contains a non-linear transformation, no assumptions about the functional form of the transformation, which makes data linearly separable, is necessary. The transformation occurs implicitly on a robust theoretical basis.

3. SVMs provide a good out-of-sample generalization if the kernel is appropriately chosen. This implies that SVMs can be robust enough, even when the training sample has some distortion or bias as in our approach occurs, because of the irregular distribution of the weeds patches in the cells. With the choice of an appropriate kernel the more similar attributes of two cells is, the higher is the value of the kernel. Thus, when classifying a new cell, the values of its attributes are compared with the ones of the support vectors of the training samples, which are more similar to this new cell. The cell is then classified according to which group it has the greatest similarity.

4. SVMs provide a unique solution, since the optimality problem is convex [31]. This represents an advantage compared to neural networks or fuzzy approaches, which can get several solutions associated to local minima, i.e. the robustness depends on the samples.

5. The main disadvantage is that SVMs cannot represent the contribution of all cells under a parametric function, because the decision function only considers the support vectors. This is common for others approaches including those that estimate some parameters, such as fuzzy clustering or Bayesian approaches [33].

Overall, based on the above reasoning and considering also the advantages reported in Section 2.2.3, SVMs become an appropriate approach for our bi-class spraying decision problem.

\section{Comparative analysis and performance evaluation}

In order to assess the validity and performance of the proposed approach, a set of 86 digital images has been used, acquired in April 2005. Experiments were conducted on an experimental farm owned by the Spanish Research Council (CSIC) located in Arganda, Madrid, i.e. in the Spanish central area where the rainfalls are not abundant.

Around a half of the images were collected on sunny days and the rest on cloudy days, with the time difference between two sets 
of corresponding images (sunny day, cloudy day) being less than 3 days, thereby ensuring that both samples corresponded to the same growth stage. Under these circumstances the digital images fundamentally represented different natural lighting conditions. All digital images were taken with a Sony DCR PC110E camera.

One important thing to clarify is that the number of images used with the Support Vector Machines approach is very low comparing with the number of images used in other previous approaches. 340 images were used in [18], 146 in [19] and 120 in [21]. This is in relation to the advantages exposed in Section 2.2.3 regarding the computational complexity and information storage. This number of images ensures the fast response of this method as well as its low computation complexity although in certain cases it is possible to obtain a weaker response compared to other methods if the training set is not well selected.

\subsection{Design of a test strategy}

Assuming that SVM involves a learning process a test strategy has been designed in order to assess the validity and performance of the proposed approach. With such purpose our test is carried out according to the following steps:

STEP 0: off-line process with $F Y_{0}$ and $F N_{0}$ cells where the number of support vectors is computed

For $n=1$ to $n=3$ do

STEP n: on-line process, make a decision for each cell through Eq. (10) obtaining $F Y_{n}$ and $F N_{n}$. Supply these cells for a new off-line process by adding these cells to the set of cells previously classified and stored in the $K B$. Obtain a new set of support vectors.

Moreover, in order to asses the performance of the attributes given by Eq. (7) three tests are defined: Test 1, Test 2 and Test 3 , where Test 1 and Test 2 use only one attribute (component) $x_{i 1}$ and $x_{i 2}$, respectively; Test 3 uses both components under the areabased vector $\mathbf{x}$. the SVM framework is applied for each test. So, Test 3 verifies the performance of the approach proposed in this paper. According to the discussion in Section 2.1.3, the attribute $x_{i 1}$ is used in Tian et al. [28] and the attribute $x_{i 2}$ is used in Ribeiro (2005). Hence, the performance of our approach is compared against such existing strategies.

\subsection{Analysis of results}

According to Eqs. (8) and (10), the decision function involves the kernel $H$, with an important role in the SVM paradigm, as described above in Section 2.2.4. Now the goal is to determine, which the best kernel $H$ is. For this purpose, we have available the set of 20 images, which is randomly selected from the full set of 86 images. This is also the set used for setting the ratio of the white area as given in Eq. (3). Hence, as described in Section 2.1.3, we have the following distribution of cells: $F Y_{0}=156$ and $F N_{0}=564$. Following the guidelines of the 10 -fold cross validation procedure [33], the cells in $F Y_{0}$ and $F N_{0}$ are randomly distributed into ten disjoint sets of similar size, i.e. each disjoint set contains 15 or 16 cells from $F Y_{0}$ and 56 or 57 from $\mathrm{FN}_{0}$.

According to the kernel $H$ chosen, the procedures for setting the free parameters is as follows:

(1) Gaussian Radial Basis Functions (RBF), $H(\mathbf{x}, \mathbf{y})=\exp [-\| \mathbf{x}-$ $\mathbf{y}||^{2} / \sigma^{2}$, we vary $\sigma^{2}$ from 1 to 10 in steps of 0.5 and for each $\sigma^{2}$ value, we estimate the decision function in Eq. (10) 10 times, each time with nine different disjoint sets, which are the training sets. The other set is used as the validation set, which allows to compute the decision error. Finally, we obtain the averaged
Table 1

Number of images and margin values ( $\tau$ ) for Gaussian Radial Basis Functions (RBF), polynomial (POL) and sigmoid (SIG)

\begin{tabular}{lllll}
\hline & STEP 0 & STEP 1 & STEP 2 & STEP 3 \\
& $20^{\mathrm{a}}$ & $42^{\mathrm{a}}$ & $64^{\mathrm{a}}$ & $86^{\mathrm{a}}$ \\
$\tau$ & & & & \\
RBF & 0.52 & 0.59 & 0.65 & 0.78 \\
POL & 0.39 & 0.40 & 0.51 & 0.57 \\
SIG & 0.33 & 0.35 & 0.42 & 0.50 \\
\hline
\end{tabular}

a \# of images.

error over the 10 estimations. We choose the minimum average error as the best performance and select the corresponding parameter as appropriate; in our experiments this occurs with for the minimum error of $22 \%$ and $\sigma^{2}=3.0$.

(2) Polynomial (POL), $H(\mathbf{x}, \mathbf{y})=\langle\mathbf{x}, \mathbf{y}\rangle^{d}$ with $\langle\mathbf{x}, \mathbf{y}\rangle$ is the inner product. Following the same procedure as above, but varying the degree $d$ from 1 to 5 we obtain the best performance for $d=2$, which is selected as the best choice. The minimum error was $31 \%$.

(3) Sigmoid (SIG), $H(\mathbf{x}, \mathbf{y})=\tanh (\rho\langle\mathbf{x}, \mathbf{y}\rangle+\gamma)$, as before, we vary $\rho$ from 1 to 10 and $\gamma$ from 1 to 5 , obtaining the best performance with the minimum error value of $36 \%$ for $\rho=2$ and $\gamma=3$.

We have applied the test strategy designed above, from STEP 0 to STEP $n$, with $n=3$ and using the three kernels (RBF, POL and SIG) with their corresponding parameters obtained as described above. At each STEP, we estimate the decision function in Eq. (10) and compute the margin $\tau$ through Eqs. (11) and (13). Table 1 displays the number of images processed and the corresponding margin values for RBF, POL and SIG. The margin $\tau$ determines which the best kernel according to the optimal hyperplane is because better decisions can be made. This implies that larger $\tau$ values indicate better performance. Hence, from results in Table 1 one can see that RBF outperforms the other two kernels. This conclusion is in accordance with the results obtained during the initial training phase for setting the free parameters. Therefore, as a final conclusion we can see that RBF provides a good generalization for achieving a robust approach as explained in Section 2.2.4.

Table 2 displays the number of cells to be sprayed $\left(F Y_{n}\right)$ and the number of cells that do not require spraying $\left(F N_{n}\right)$. As before, the new cells are added to the old ones. In STEP 0 they are manually selected (this implies that the same number is used for each Test) and then for STEPs 1-3 this selection is carried out by the SVM classifier. For each Test the total number of support vectors is also displayed. The small number of support vectors confirms the second advantage exposed in Section 2.2.3.

Table 2

Number of images, number of cells to be or not sprayed and support vectors for each test.

\begin{tabular}{lrrrr}
\hline & STEP 0 & STEP 1 & STEP 2 & STEP 3 \\
& $720^{\mathrm{a}}$ & $1512^{\mathrm{a}}$ & $2304^{\mathrm{a}}$ & $3096^{\mathrm{a}}$ \\
Test 1 & & & & \\
$F Y_{\mathbf{n}}$ & 156 & 473 & 668 & 836 \\
$F N_{n}$ & 564 & 1039 & 1636 & 2260 \\
Support vectors & 44 & 51 & 62 & 77 \\
Test 2 & & & & \\
$F Y_{n}$ & 156 & 507 & 743 & 901 \\
$F N_{n}$ & 564 & 1005 & 1561 & 2159 \\
Support vectors & 46 & 58 & 63 & 89 \\
Test 3 & & & & \\
$F Y_{n}$ & 156 & 414 & 598 & 724 \\
$F N_{n}$ & 564 & 1098 & 1706 & 2372 \\
Support vectors & 22 & 35 & 47 & 68 \\
\hline
\end{tabular}

\footnotetext{
a Number of cells.
} 


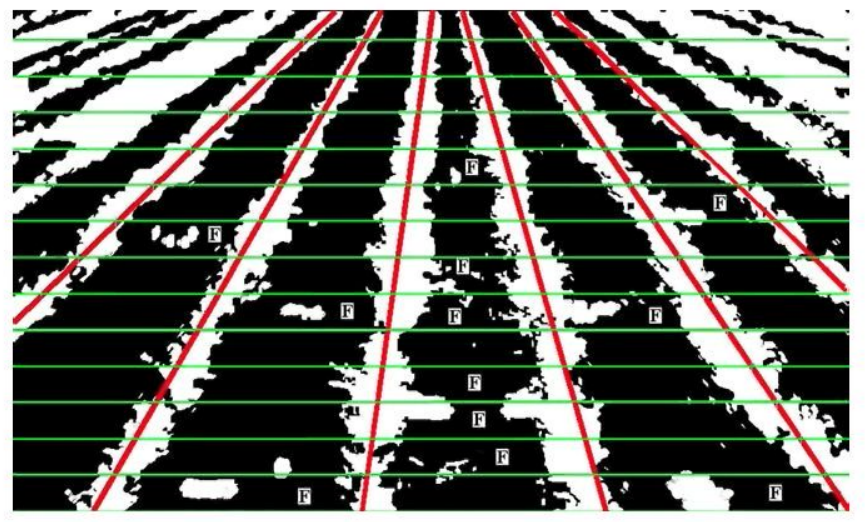

Fig. 3. Labelled image with the cells "F" to be sprayed.

The results of the comparison between the expert human criterion and the different Tests analysed are based on the following values:

True Spraying (TF): number of cells correctly identified to be sprayed.

True No Spraying (TN): number of cells that do not require spraying correctly detected.

Folse Spraying $(F F)$ : number of cells that do not require spraying but identified as cells to be sprayed by the method.

Folse No Spraying $(F N)$ : number of cells requiring spraying that they are identified by the method as cells that do not require spraying.

Traditionally, from these four quantities several measures have been used for classification [34]. The best ones are the following that combine all four values:

(1) The correct classification percentage: $\mathrm{CCP}=(T F+T N) /(T F+$ $F F+T N+F N)$

(2) The Yule coefficient: Yule $=\mid((T F) /(T F+F F))+((T N) /(T N+$ FN)) -1

Fig. 3 shows an image, which is segmented and processed according to the method described in this paper. The cells labelled with the symbol " $F$ " are to be sprayed based on the SVM decision making.

Table 3 shows the results in terms of the correct classification for the three tests. The CCP and Yule scores are computed for the set of $\mathbf{8 6}$ images and $\mathbf{3 0 9 6}$ cells. Larger score values indicate better performance.

From results in Table 3 it can be inferred that the best performance is achieved by the Test 3 in STEP 3. This means that the use of two attributes (weed coverage and weed pressure in Test 3 ) improves the performance against the use of a unique attribute (Tests 1 and 2). An improvement in the performance is also achieved as the learning progresses, i.e. during the STEP 3.

Table 3

CCP and Yule score values for the tests and steps.

\begin{tabular}{lllll}
\hline & \% & STEP 1 & STEP 2 & STEP 3 \\
\hline \multirow{2}{*}{ Test 1 } & CCP & 63 & 67 & 66 \\
& Yule & 55 & 65 & 57 \\
\multirow{2}{*}{ Test 2 } & CCP & 64 & 71 & 71 \\
& Yule & 59 & 62 & 66 \\
Test 3 & CCP & 73 & 80 & 85 \\
& Yule & 66 & 76 & 80 \\
\hline
\end{tabular}

\section{Conclusion}

A new approach for detecting a special kind of weeds: avena sterilis. This weed appears mixed in the crop fields and irregularly distributed. Moreover, the weeds and the crops have similar spectral signature and texture. This means that its identification in a cereal crop field is s difficult task. It has been designed a new strategy which involves two steps: segmentation and decision making.

The segmentation is based on a combination of basic processing techniques. The decision making is carried out through the well-tested SVM framework, which is a suitable approach when the training increases. It also offers an optimum performance in terms of computational complexity and memory requirements. The combination of the weed coverage and weed pressure attributes improves the performance of the approach as compared with the use of these attributes separately. An important issue that is to be analysed in future works is the robustness of the proposed approach against illumination variability. This is because the robot-tractor where the system is installed goes in a direction and its opposite, i.e. the illumination coming from the sun varies.

\section{Acknowledgement}

Part of the work has been performed under project AGL-200506180-C03-03.

\section{References}

[1] D.C. Cloutier, ]. Ascard, European Weed Research Society, Proceedings, in: Proc 6th European Weed Research Society (EWRS). Workshop on Physical and Cultural Weed Control, Lillehammer, Norway, 2004, pp. 8-10.

[2] A.]. Pérez, F, López, J.V. Benlloch, S. Christensen, Colour and shape analysis techniques for weed detection in celeral fields, Computers and Electronics in Agriculture $25(2000)$ 197-212.

[3] M.J. Aitkenhead, I.A. Dalgetty, C.E. Mullins, A.].S. McDonald, N.J.C. Strachan, Weed and crop discrimination using image analysis and artificial intelligence methods, Computers and Electronics in Agriculture 39 (2003) 157-171.

[4] P.M. Granitto, P.F. Verdes, H.A. Ceccatto, Large-scale investigation of weed seed identification by machine vision, Computers and Electronics in Agriculture 47 (2005) 15-24.

[5] C.M. Onyango, JA. Marchant, Segmentation of row crop plants from weeds using colour and morphology, Computers and Electronics in Agriculture 39 (2003) 141-155.

[6] H.T. Søgaard, H.J. Olsen, Determination of crop rows by image analysis without segmentation, Computers and Electronics in Agriculture 38 (2003) 141-158.

[7] C.C. Yang S.O. Prasher, ].A. Landry, H.S. Ramaswamy, Development of an image processing system and a fuzzy algorithm for site-specific herbicide applications, Precision Agriculture 4 (2003) 5-18.

[8] R. Gerhards, H. Oebel, Practical experiences with a system for site-specific weed control in arable crops using real-time image analysis and GPS-controlled patch spraying, Weed Research 46 (2006) 185-193.

[9] K.R. Thorp, LF. Tian, A review on remote sensing of weeds in agriculture, Precision Agriculture 5 (2004) 477-508.

[10] J. Barroso, C. Fernández-Quintanilla, C. Ruiz, P. Hernaiz, LJ. Rew, Spatial stability of Avena sterilis ssp. Ludoviciana populations under annual applications of low rates of imazamethbenz, Weed Research 44 (2004) 178-186.

[11] L. Radics, M. Glemnitz, J. Hoffnann, G. Czimber, Composition of weed floras in different agricultural management systems within the European climatic gradient, in: Proc. 6th European Weed Research Society (EWRS). Workshop on Physical and Cultural Weed Control, Lillehammer, Norway, 2004, pp. 58-68.

[12] B. Bacher, Weed density estimation from digital images in spring barley, $\mathrm{PhD}$ thesis, Dpt. Agricultural Sciences, Section of AgroTechnology, The Royal Veterinary and Agricultural University, Denmark, 2001.

[13] M.J. Kropff, J. Wallinga, L.A.P. Lotz, Modelling for precision weed management, in: Precision Agriculture: Spatial and Temporal Variability of Environmental Quality, Wiley, Chichester, 1997, pp. 182-204.

[14] B. Astrand, A.]. Baerveldt, An agricultural mobile robot with vision-based perception for mechanical weed control, Autonomous Robots 13 (2002) 21-35.

[15] A. Tellaeche, X.P. Burgos-Artizzu, G. Pajares, A. Ribeiro, On combining Support Vector Machines and Fuzzy $\mathrm{K}-$ Means in vision-based precision agriculture, in; International Conference on Computer, Electrical and Systems Science, and Engineering (WASET 2007), Volumen 22 Julio 2007, 2007, ISBN 978-97501752-1-3, pp. 33-38 (CD-ROM).

[16] A. Tellaeche, X.P. Burgos-Artizzu, G. Pajares, A. Ribeiro, A vision-based classifier in precision agriculture combining Bayes and Support Vector Machines, in: IEEE International Symposium on Intelligent Signal Processing (WISP 2007), Conference Proceedings Book, 2007, ISBN 1-4244-0829-6, pp. 425-430, IEEE Catalog Number: 07EX1620 (CD-ROM). 
[17] A. Tellaeche, X.P. Burgos-Artizzu, G. Pajares, A. Ribeiro, A vision-based hybrid classifier for weeds detection in precision agriculture through the Bayesian and Fuzzy k-Means paradigms, in: 2nd International Workshop on Hybrid Artificial Intelligence Systems (HAIS). Innovations in Hybrid Intelligent Systems. Advances in Soft Computing, vol, 44, Springer-Verlag, Berlin, Heidelberg, 2007, ISBN 978-3-540-74971-4, pp. 72-79.

[18] A. Tellaeche, X.P. Burgos-Artizzu, G. Pajares, A. Ribeiro, A vision-based method for weeds identification through the Bayesian decision theory, Pattern Recognition 41 (2) (2008) 521-530.

[19] A. Tellaeche, G. Pajares, A. Ribeiro, X.P. Burgos-Artizzu, A new vision-based approach to differential spraying in precision agriculture, Computers and Electronics in Agriculture 60 (2) (2008) 144-155.

[20] A. Tellaeche, Técnicas inteligentes basadas en percepción visual aplicadas a la agricultura de precisión, Doctoral Thesis, UNED, 2008.

[21] G. Pajares, A. Tellaeche, X.P. Burgos-Artizzu, A. Ribeiro, Design of a computer vision system for a differential spraying operation in precision agriculture using Hebbian learning, IET Computer Vision 1 (3-4) (2007) 93-99.

[22] L.F. Tian, D.C. Slaughter, Environmentally adaptive segmentation algorithm for outdoor image segmentation, Computers and Electronics in Agriculture 21 (1998) 153-168.

[23] P.L. Rosin, E. Ioannidis, Evaluation of global image thresholding for change detection, Pattern Recognition Letters 24 (2003) 2345-2356.

[24] J. Kapur, P. Sahoo, A. Wong, A new method for gray-level picture thresholding using the entropy of the histogram, Computer Vision Graphics Image Processing 29 (3) (1985) 273-285.
[25] J. Billingsley, M. Schoenfisch, The successful development of a vision guidance system for agriculture, Computers and Electronics in Agriculture 16 (1997) 147-163.

[26] R.C. Gonzalez, R.E. Woods, S.L. Eddins, Digital Image Processing using Matlab, Prentice Hall, New York, 2004.

[27] R.C. Gonzalez, R.E. Woods, Digital Image Processing, Addison-Wesley, New York, 1993.

[28] L. Tian, J.F. Reid, J.W. Hummel, Development of a precision sprayer for site-specific weed management, Transactions of the American Society of Agricultural Engineers (ASAE) 42 (4) (1999) 893-900.

[29] A. Ribeiro, C. Fernández-Quintanilla, J. Barroso, M.C. García-Alegre, Development of an image analysis system for estimation of weed, in: Proc. 5th European Conf. on Precision Agriculture (5ECPA), 2005, pp. 169-174.

[30] V. Cherkassky, F. Mulier, Learning from Data: Concepts, Theory and Methods, Wiley, New York, 1998.

[31] V.N. Vapnik, The Nature of Statistical Learning Theory, Springer-Verlag, New York, 2000 .

[32] T. Evgeniou, M. Pontil, T. Poggio, Statistical learning theory: a primer, International Journal of Computer Vision 38 (1) (2000) 9-13.

[33] R.O. Duda, P.E. Hart, D.G. Stork, Pattern Classification, John Willey and Sons, New York, 2001.

[34] P. Sneath, R. Sokal, Numerical Taxonomy: The Principle and Practice of Numerical Classification, W.H. Freeman, 1973. 